Kalpa Publications in Computing
Volume 6, 2018, Pages 67-82
WDAM-2017. Workshop on Data Analysis in Medicine

\title{
Modeling Framework for Medical Data Semantic Transformations
}

\author{
Alexander Vodyaho ${ }^{1}$, Nataly Zhukova ${ }^{1,2}$, Dmitry Kurapeev ${ }^{3}$ and Mikhail Lushnov ${ }^{3}$ \\ ${ }^{1}$ Saint-Petersburg Electrotechnical University “LETI”, Saint-Petersburg, Russia \\ ${ }^{2}$ ITMO University, Saint-Petersburg, Russia \\ ${ }^{3}$ Federal Almazov North-West Medical Research Center, Saint-Petersburg, Russia \\ aivodyaho@mail.ru, nazhukova@mail.ru
}

\begin{abstract}
Nowadays in the domain of modern information technologies there is an evident trend of wide usage of intelligent information systems (IS), where processing of incoming data is based on multiple semantic-oriented transformations. Model Driven Engineering approach is widely used for intelligent IS development. The effectiveness of this approach is defined by availability for developers sufficient number of domain oriented models, which describe the classes of solutions and suggest effective tools for model transformation. In the paper a model driven approach for development of intelligent IS is described. The main idea of the suggested approach is an implementation of semantic oriented transformations. This approach was used by the authors for building real IS for different subject domains. The example of practical usage of the suggested approach for medical IS development is described.
\end{abstract}

Keywords: model driven approach for domain specific intelligent information system development, measurements processing and analyses, modeling framework, models semantic transformations

\section{Introduction}

In the sphere of modern information technologies one can observe two prominent trends. The first one is allocating all surrounding thing with intelligence, the second one reflects the idea of creating a global linked data network. The first trend is realized in the frames of such concepts as pervasive 
computing, ambient intelligence aka the Internet of Things [1], the second one - within the semantic web paradigm [2].

By now in the real world the majority of technical and natural objects have embedded and / or external devices which can gather and provide data about their own state and the state of the environment. In this case data by the most part are raw streams of multi-modal measurements that contain time series, separate measurements, qualitative data, texts, images, sounds etc. The amount of collected data crushes recently used approaches for expert oriented manual data processing, analysis and linking (DPAL).

There is a number of contradictions in the list of requirements to modern intelligent IS. On one hand, modern intelligent IS become more and more complex. In order to develop such IS it is necessary to have a lot of high skill developers. On the other hand, in order to enlarge the scope of intelligent IS usage it is necessary to minimize the total cost of ownership (TCO). An evident solution of this problem is the usage of effective development methodologies and tools such as Model Driving Engineering (MDE). The effectiveness of MDE approach is defined by availability for developers domain oriented models and frameworks, which describe the classes of solutions and effective tools for model transformation. MDE assumes the use of hierarchy of models, which describe raw data processing, information or knowledge processing. For practical use it is necessary to have specialized models applicable to specific subject domains.

Processing results of measurements from technical objects. The results of a detailed analysis of existing data, information and knowledge (DIK) models in the domain of data processing made by the authors show that: i) models have been implemented only within research projects; ii) models and transformations have strong restrictions on the scope of application, only few tasks are in scope; iii) models are not defined formally, often contain a considerable number of significant contradictions; iv) models are diverse, though they have noticeable intersection; v) as a rule models are used only once even in the frames of single project.

In the paper a domain specific knowledge based model driven approach for building intelligent IS for processing results of measurements is discussed. A new approach for building DIK models for measurements processing and analysis is proposed. It allows define models at the level of objects. In order to support a high level of abstraction it is suggested to develop and use a new modeling framework. In the paper by the term framework a specialized framework of the subject domain level that is intended to solve data processing and analysis problems is assumed. It is an architectural model driven framework that includes an ordered set of mathematically defined DIK models and set of transformations sufficient for realization necessary transitions between models.

Nowadays there are a big number of technical and natural objects which already have embedded and / or external devices for gathering and providing data about their own state and state of the environment. Data is raw streams of multimodal data that contain time series, separate measurements, qualitative data, texts, images, sounds etc. It can be linked with the source object as well as with other connected or separate objects and systems. Amount of collected data becomes too big to be processed by experts manually. There is a number of convincing examples that show benefits of semantic technologies application which can support business process that require data processing and analyses in separate applied subject domains $[3,4]$.

The semantic oriented data processing can show good results only in the case when there is enough of available DIK [5] about the target subject domain and about the context of the solved tasks. Semantic oriented processing is a sequence of data transformations based on models and methods of the artificial intelligence, that are implemented within semantic technologies.

The main obstacles of the semantic oriented data processing and analyses are the following: i) complexity of DIK models and their interrelations; ii) complexity of operations for DIK models transformations; iii) problems with models population. 
The problem of the data processing and analyses, DIK models formalization, systematization and providing them with capabilities to build and support a linked data cloud brought to decision to develop a modeling framework.

In common sense the framework is a real or conceptual structure intended to serve as a support or guide for building of something that expands the structure into something useful [6] that can be defined at different levels of abstraction. In the paper the framework is a subject domain level framework which is to be used for solving data processing and analyses problems. It is a model driven framework that includes an ordered set of mathematical defined DIK models and set of transformations sufficient for transitions between models. In the second section of the paper semantic technologies application in the domain of data processing is discussed. The third and the fourth sections contain the proposed DIK models and descriptions of semantic transformations. Business logic of the IS that supports model based measurements data transformations are discussed in section five. In the sixth section the framework for designing systems that use semantic transformations of measurements data is presented. In the last section an example of practical usage of suggested approach is discussed.

\section{Semantic gaps in measurement data processing and analyses technologies}

Measurement data processing and analyses can be realized on a number of levels. For describing processing on different levels various models, approaches, algorithms and technologies are used. So, one can observe essential semantic gaps even between neighboring levels. The conceptual DPAL model is given in Fig. 1. Real world objects and systems called target systems (TS) are sources of raw data. Sensors, mounted on the objects, measure values of diverse internal and external parameters, gather and pack them. Packed measurements (M) are transferred to consumers (C) using telemetric information systems (TMI S). The examples of the parameters are temperature, pressure. The consumers of the data can be both end users and external IS.

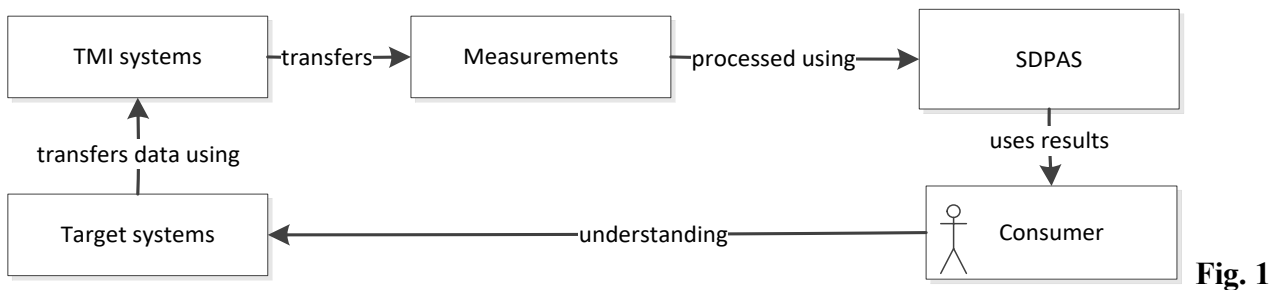

Raw data is useless for the majority of the consumers as it is not interpretable and cut off from their information space. Semantic data processing and analyses systems (SDPAS) are applied to transfer raw data into linked information and knowledge. They have to represent data in the form interpreted by the machines and understandable by humans.

The overall list of the high level transformations assumed by the conceptual model includes following items: i) "measuring" - transformation of physical objects parameters (OP) values to digital measurements (DM) using sensors (S) of the target system (TS): ii) "packaging" - transformation of digital measurements to raw data streams (DS) using telemetric information systems (TMI systems); data streams are transferred to data centers for further processing and analyses: iii) "processing and analyses" - transformation of raw data streams to formalized data, DIK interpretable by the consumers using SDPAS: iv) "understanding" - transformation of DIK model by the consumers to acquire the target information about the physical object.

Some of the transformations depend on the analyzed objects properties and features of the TMI Systems. These transformations can be formalized. Measurements processing procedures can be described in terms of the subject domain of data analyses. A poorly formalized process that completely 
depends on the consumers is the transformation of DIK model build by SDPAS to the information about the physical object. This semantic gap is considered below.

Many authors have indicated this problem [7], but general solution was not found. In this paper it is suggested to use domain specific model driven approach to SDPAS development.

\section{Model driven representation of measurements processing and analyses}

The sequence of transformations from the modeling point of view is given in Fig 2. Model driven approach for measurements processing and analyses has been proposed and proved its efficiency [8].

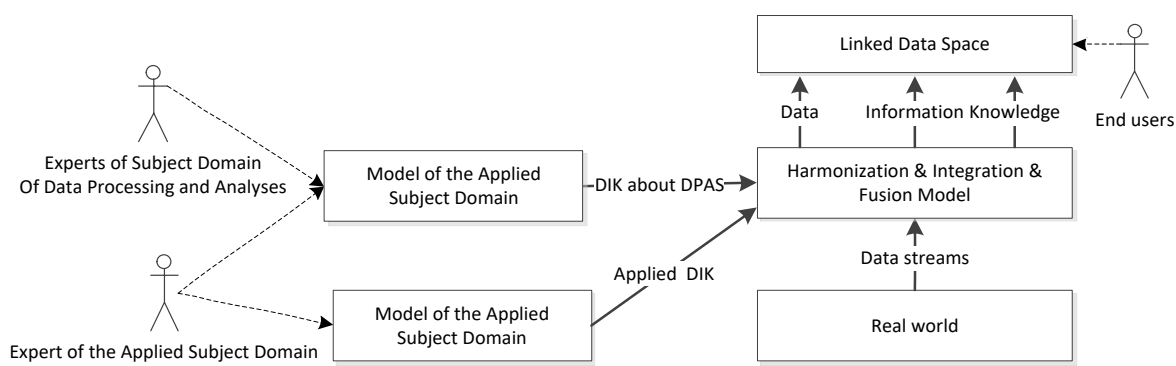

Fig. 2

At the stage of DPAS usage in real world provides structured data streams which contain results of operational measurements. With application of a set of formalized operations dynamic and static data and information about the real world objects is processed and analyzed. Dynamic data are raw data streams of results of measurements collected during periods of objects functioning. The static data is apriori information provided by the applied subject domain.

According to considered sequence of the transformations given in Fig. 2 in general case 9 key models are required. Two of them are models of real world objects - target systems (M TS) and TMI systems (M TMI S), one describes measurements of target systems (M M), another one is the IS (M DPAS) oriented on measurements processing. A general model is associated with each of the models. GM TS (general model of target systems), GM TMI (general model of TMI systems) relate to the models of the applied subject domain, GM M (general measurement model) - to the domain of data processing and analyses, SM DPAS (static model of DPAS) and DM DPAS (dynamic model of DPAS) are general models that form the base of the IS (Fig.3).

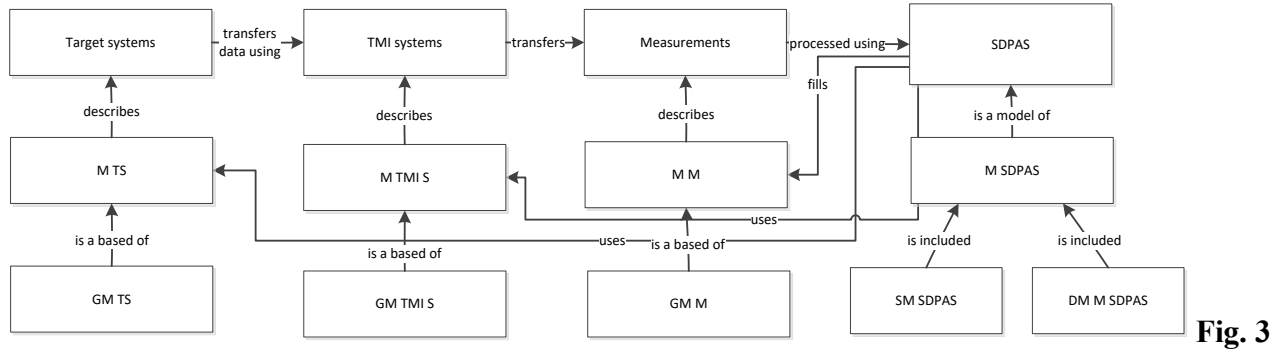

The process of measurements transformations to linked data cloud assumes that the models of existing objects are build according to the general models. The system deals with the formalized models, not with the objects. Such models should have three principle features: i) reflect the objects of the real world; ii) represent information and knowledge in the form that the can be interpreted and consumed 
by computers; iii) define general models of real world objects that can be built and updated automatically.

The applicability of the models depends on the features of the general models. Thus, the key task is to define the structure and the contents of the general models. It is strongly required that general models should be simple enough to be understandable and interpretable by the experts of subject domain or, at least, can be represented with a number of simple views and must support transparent projections of general models to real world objects models.

GM M are proposed in [8]. The TMI are typical and well formalized. The general model of TS are discussed in the next section.

\section{General Information Models of Target Systems}

The general model of the target system that represents the real world natural or artificial objects contains structural model, logical model, mathematical model, experimental model, statistical model (Fig. 4).

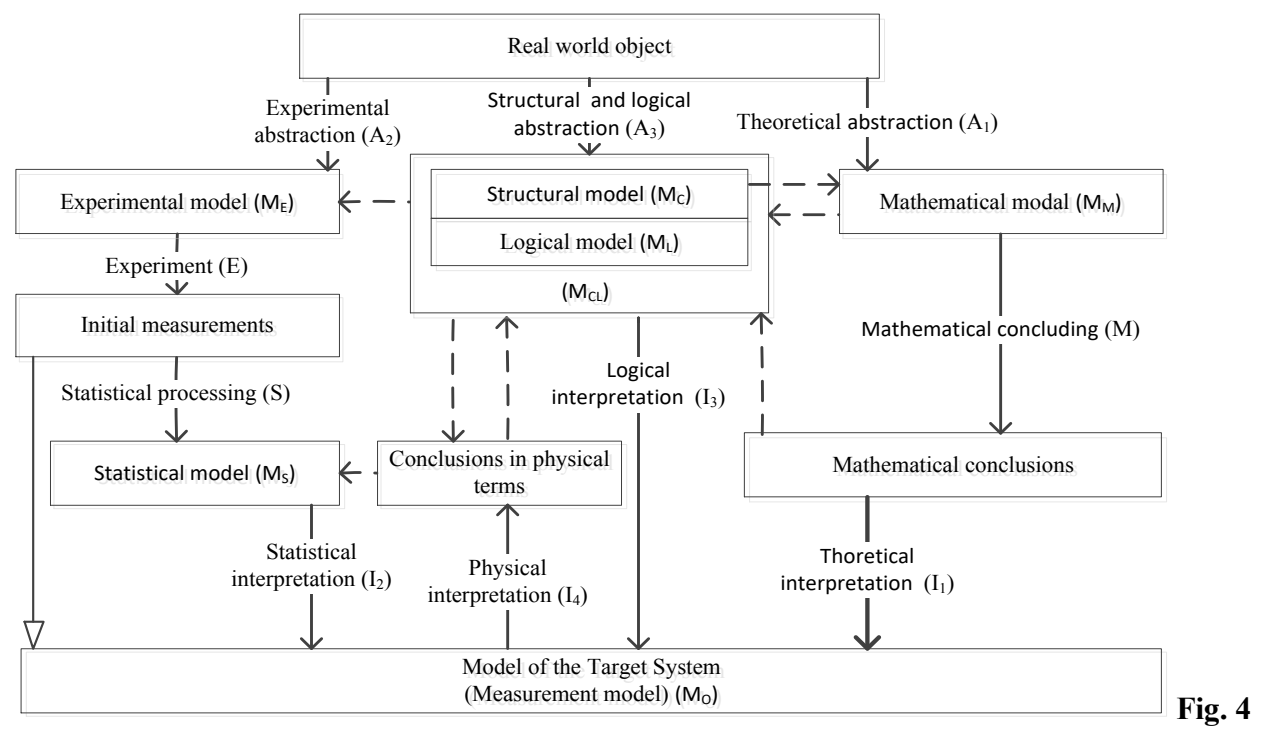

The general model supports three types of transformations: abstraction, interpretation, and operations DIK conversion. To the latter group of transformations refer experiment $(E)$, mathematical concluding $\left({ }_{M}\right)$, statistical processing $\left({ }_{S}\right)$. Abstraction transformations $\left({ }_{A}\right)$ allow describe initial object from four points of view: mathematical, structural, logical and experimental. For building model of a target system interpretation $\left(_{I}\right)$ of the results of mathematical concluding, statistical processing, results of logical operations are carried out.

The elements of the model and their brief descriptions are given in Table 1. 
Table 1. The structure of the TS model

\begin{tabular}{|l|l|l|l|}
\hline Id & Model name & Purpose of the model & Representation form \\
\hline$M_{M}$ & $\begin{array}{l}\text { mathematical } \\
\text { model }\end{array}$ & $\begin{array}{l}\text { mathematical description of } \\
\text { states and behavior of an object }\end{array}$ & $\begin{array}{l}\text { functional dependenc- } \\
\text { es }\end{array}$ \\
\hline$M_{C}$ & $\begin{array}{l}\text { structural mod- } \\
\text { el }\end{array}$ & $\begin{array}{l}\text { description of the physical } \\
\text { elements which are a part of an } \\
\text { object, their interrelations }\end{array}$ & $\begin{array}{l}\text { sets, relations between } \\
\text { elements in the set }\end{array}$ \\
\hline$M_{L}$ & logical model & $\begin{array}{l}\text { description of the logical ele- } \\
\text { ments which are a part of an } \\
\text { object, their interrelations }\end{array}$ & logical expressions \\
\hline$M_{E}$ & $\begin{array}{l}\text { experimental } \\
\text { model }\end{array}$ & $\begin{array}{l}\text { description of the real object in } \\
\text { the context of solving one / } \\
\text { several tasks }\end{array}$ & $\begin{array}{l}\text { formalized qualitative } \\
\text { and quantitative in- } \\
\text { formation about object } \\
\text { operating conditions }\end{array}$ \\
\hline$M_{S}$ & $\begin{array}{l}\text { statistical mod- } \\
\text { el }\end{array}$ & $\begin{array}{l}\text { statistical characteristics of the } \\
\text { state and behavior of an object, } \\
\text { dynamics of their change }\end{array}$ & $\begin{array}{l}\text { time series, separate } \\
\text { values }\end{array}$ \\
\hline
\end{tabular}

\section{Architectural Approach to the Business Logic Description of Measurement Data Processing Systems}

Information models considered above allow build comprehensive formalized descriptions of real world objects that are based on measurements and results of their processing. In order to build such description the list of transformations is defined. Both object models and transformations are abstract. Different kinds of technologies end services can be used. It is reasonable to select them taking into account the requirements to each task or group of tasks. So the task is to define a flexible approach for abstract models implementation using the variety of existing software solutions.

The suggested approach contains two principle steps:

- description of the models from several architectural viewpoints (VP);

- definition of a taxonomy for elements of each VP.

From these elements end systems and executable processes can be generated.

An architecture of a system can be described as a set of the following viewpoints (VP): systems VP, capability VP, models VP, objects VP, DIK VP, process (behavior) VP, services VP, projects VP, standards VP, all VP.

Systems VP describes a system in terms of elements and connections, capability VP - in terms of functions to be realized, models VP - in terms of used models, objects VP gives object oriented descriptions of the system, DIK VP describes used data, information and knowledge formats, processes VP describes system in terms BPs processes to be implemented, services VP describes the system as a set of services and scripts (business processes), projects VP is a description of the system from the point of view of development, standards VP - from the point of view of standards to be used and all VP integrates all other VP.

In the context of well known architectural model [6] all VP can be divided into 4 groups: conceptual VPs, logical VPs, Process VPs and Physical VPs. Process VPs describe dynamics of system operation. 


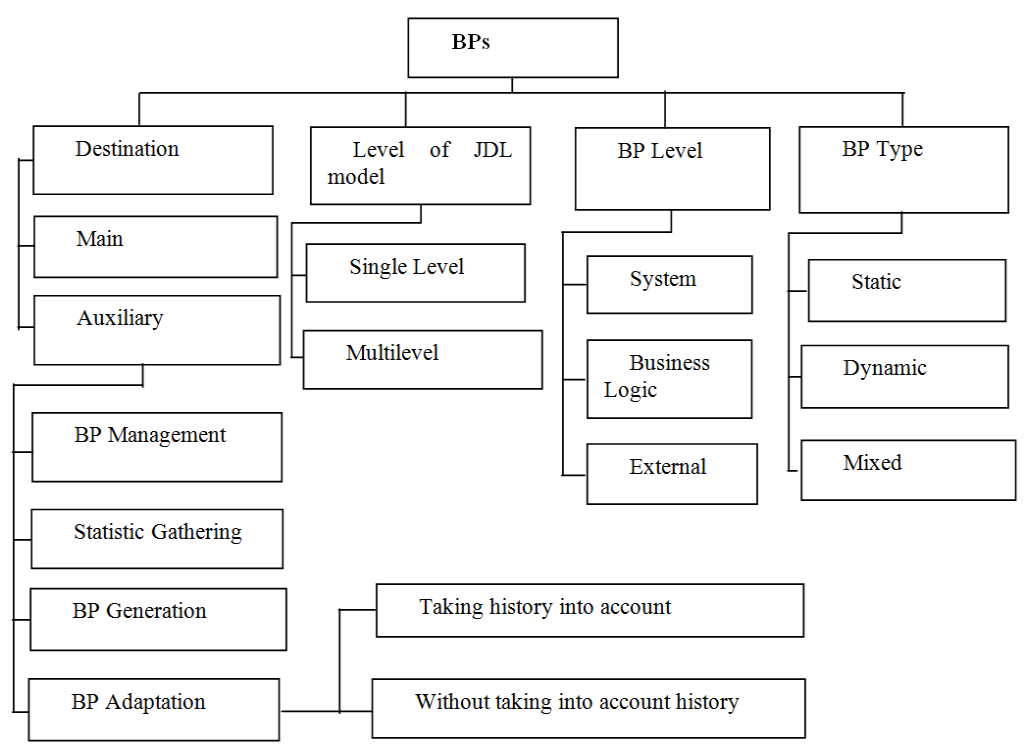

Fig. 5

Operation of a system is described in terms of business processes (BPs) description languages such as UML, IDEF etc. Business logic can be defined as a set of BPs which are realized inside the system. BPs taxonomy is shown in Fig. 5.

BPs can be classified according to destination, level of JDL model [8], BP level and BP type. According to provided destination it is possible to distinguish the main and auxiliary BP. The main BPs implement business logic, auxiliary BPs can be divided into management BPs, BPs, responsible for system statistics gathering and processing, BPs which are responsible for generation of other BPs and BPs for realization mechanisms of adaptation. The latter one can use history information. BPs can cover one or several levels of JDL model. BPs can operate on the system level, on the level of business logic or the external level. The latter is used for interaction with external systems. BPs can be formed in static, in dynamic (run time) or in mixed mode, for example, with the help of build-in business rules [9]. Classification of possible approaches to BPs implementations is shown in Fig. 6. 


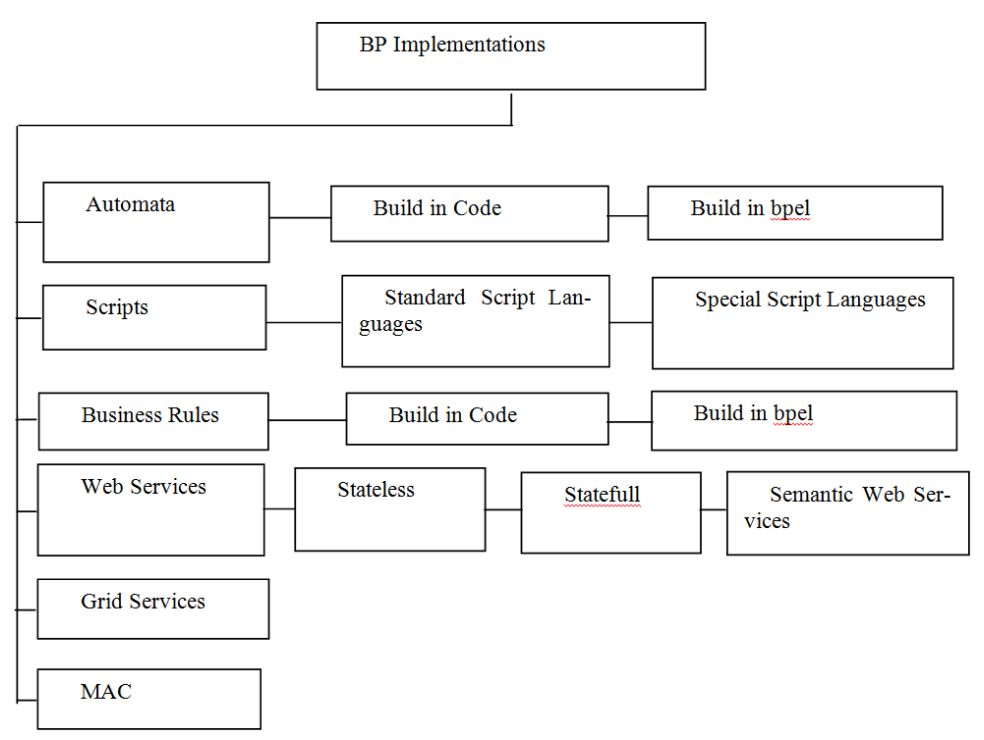

Fig. 6

It is possible to use different architectural solutions for SDPAS building, such as Web Services (stateless, stateful or semantic), microservices [10], business rules, Grid Services or multi agent systems (MAC). Service oriented solutions (SOA) [10] allow receive agile solutions. Both Web services and microservices solutions can be used. While using Web services based on architectures for generation of BP in dynamics it is possible to use at least two options: Semantic Web services or business rules (build in code or build in bpel). While realizing microservices solutions one can use automata models for generation managing scripts in dynamic [11]. This solution is rather simple and effective when SOAP is not used.

\section{Architectural Framework for System of Semantic-Oriented Measurements Data Transformations}

To implement the described approach an architectural framework (AFW) for supporting a process of architectural design of IS for processing information from complicated artificial and nature systems was developed. The developed AFW uses visual environment [12]. The main ideas which are realized in the AFW are the following: i) Process model is used as a base model for processing measurements in target systems, ii) Wide usage of mechanism of dealing with knowledge both for AFP and target system. iii) Usage of COTS (Commercial off-the-shelf) solutions. Suggested AFW uses service oriented architecture and is implemented on the base of enterprise service bus (ESB [10]). It can be deployed as a standalone system or used as a module in standard system for development support, such as Enterprise Architect [13]. AFW can be integrated with other tools such as Eclipse, search engines, ontology editors, etc. It can be used for development of models, services and scripts that realize BPs, which are to be executed in IS where it is necessary to process measurements information from complex artificial and natural objects on different levels of abstraction.

The suggested framework has a number of common features with well known Zachman framework, which can be positioned as general purpose architectural framework. Suggested framework can be positioned as domain oriented architectural framework and it is to be used for designing only enough narrow class of IS - intelligent systems for processing results of measurements. In comparison with Zachman framework it is more effective but less universal. Principally suggested framework can 
be use as a prototype for building domain specific frameworks for other subject domains, but in this case it must be populated with different models.

The structure of the AFW is shown in Fig. 7. It includes following main elements: Builder (B), storage, enterprise service bus (ESB), internal services (Services), B2B services and Import-Export subsystem.

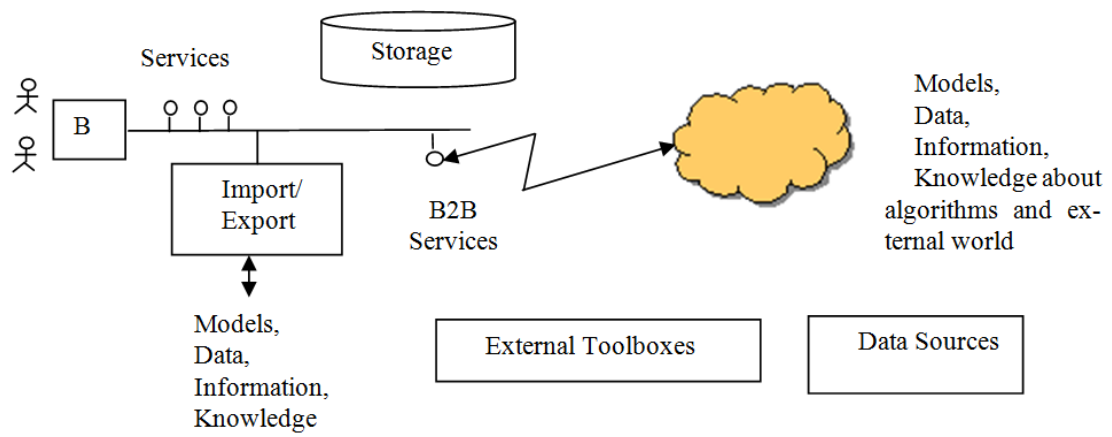

Fig. 7

B is a key element of the AFW which includes business logic and a number of screen forms. A user can build models, services and BP. For building BP graphical internal script language is used. An interpreter of the internal script language is a part of B. The second key element of the AFW is the ontology of models, methods, algorithms, services and scripts. The ontology and data are placed in the storage. ESB is used as a services integration tool.

The AFW is implemented as a number of internal and B2B (external) services. Internal services are Web services (microservices can be also used). The list of internal services includes following services: infrastructure services (InfrS), business services (BS), engines. InfrS are low level services that support separate operations of harmonization, integration and fusion [15]. Data and ontology access services one can consider as InfrS. BS are high level services that are formed from low level services with the help of scripts. Following engines are used inside AFM: bpel engine, business rule engine. Some additional engines can be also used in future such as interpreters of standard script languages and Semantic Web Service engine. B2B services are used for interaction with external toolboxes different data sources and external services. They are also used for gathering information about models and algorithms from different sources including Internet. From the point of view of a user, engine and BP look like services. Import/Expert subsystem, by the most part, is used for import and export of UML models and ontologies.

Main stakeholders of the AFW are Architect, Systems Analyst, Sponsors and Administrator. An Analyst works with models, algorithms and services and can create, edit, save and delete rights. Also he or she can form and run scripts. An Architect mainly works with models and scripts and can create, edit, save and run scripts. He or she also can work with services but as a rule in read only mode. Sponsor is a person with restricted (guest) rights who can only add models, algorithms and services in the knowledge base. Nowadays Sponsor is a human person, but it is very desirable to substitute a human by a service, that could form knowledge bases automatically. Main function of this service is intelligent search in variety of different sources of information and knowledge including Internet. This service would be very useful when it is necessary to build models of external world for new subject domain. System administrator gives access rights to users and realizes monitoring functions.

The AFW incorporates a set of model transformation services which allow effectively support the process of measurement conversions on the way from unstructured data to well defined highly formalized knowledge store is represented by a process chain (elements of process view) to analyze data blocks at each of the stages (data view). The following types of transformations are supported: i) real 
world object $\rightarrow$ observations and measurements; ii) raw measurement data (values and measuring units) $\rightarrow$ storage of the data into establishment database; iii) measurement data stored in a nonhierarchical database of establishment $\rightarrow$ extraction and parsing process pipeline, measurement analysis and preprocessing; iv) semi structured preprocessed measurements and measuring units $\rightarrow$ measurement analysis and processing, storing into object database corresponding the domain object model structure; v) stored semi-structured information on measurements $\rightarrow$ matching with ontological model; vi) formalized measurement information $\rightarrow$ reference and thesauri-driven measurement information processing; vii) structured triple set of measurement data in RFD format $\rightarrow$ storing triples into triple store to form a knowledge graph.

Described above AFW can be concided as a core or metaframework. It can be used for creation subject domain specific AFW, which can se used for real systems development. The procedure of subject domain specific AFW assumes tuning the list of services, adding subject domain specific services, development subject domain specific ontologies, etc. It was done by the authors for a number of subject domains including medicine.

\section{Case Study. Semantic Medical Data Analyses System}

The developed medical AFW is to be used by the doctors for solving real doctor' problems. Developed Semantic Medical Data Analyses System (SMDA) is an intelligent agile IS, which is used to analyze the results of echocardiography for various diseases but additional features can be easily added without system redesign.

The system has 2 key features: i) system is based on semantic technologies; ii) key system elements are COTS (Commercial off-the-shelf) products.

The system has 3 layer architecture: i) layer of DIK storage, where raw data, ontologies and logical rules are stored; ii) layer of service and business process, which encapsulates services for data models processing, BPEL engine, logic inference engine, business rule engine and the library of algorithms for data processing and analysis; iii) GUI layer, which includes a number of specialized UI forms for measurements cognitive representation and analysis, and special editors which allow build and edit dictionaries and thesauri, business rules and business processes.

The realization is based on the semantic platform that is conceded as a component of knowledge storage base and queries, production rule-based inference and knowledge base display and analysis that are represented by the following components: i) BlazeGraph ${ }^{1}$ is conceded as a scalable and flexible triple store and SPARQL-endpoint for search and inference and a high-performance graph database platform with support of RDF for knowledge data-set upload with a support of up to 50 billion edges; ii) Metaphactory ${ }^{2}$ is conceded as a product providing powerful solutions and numerous services for describing, querying and interchanging graph-based data, as well as a user-oriented open platform to visualize and interact with knowledge graphs; iii) Ontodia ${ }^{3}$ is conceded as a simple and free of charge online OWL and RDF diagramming tool to represent a knowledge base with no programming involved; iv) Apache Jena ${ }^{4}$ is conceded as a free and open source framework for Java to build Semantic Web and Linked Data applications based on ontologies and elements of inference (needs to be supported with powerful inference engines based on production logic such as Drools).

\footnotetext{
${ }^{1} \mathrm{https}: / /$ www.blazegraph.com/product/

${ }^{2} \mathrm{http}: / /$ www.metaphacts.com/product

${ }^{3} \mathrm{http}: / /$ www.ontodia.org

${ }^{4} \mathrm{https}: / /$ jena.apache.org
} 
Domain platform including a wide range of tools from initial data display tools (medical information systems, MIS) to medical data processing (domain-specific and internal intelligent services) to provide a processing pipeline from input as an API request to user interface as a result of all processing stages requested by a user.

All of the raw, preprocessed and semantically processed data are stored in a lower layer in data storage of four data state levels: unstructured data, preprocessed semi-structured data, data covered by ontologies and a rule base. These are unstructured raw data storages, semi structured DB Caché ${ }^{5}$ and processed data storages and ontology storages of domain area.

The other part of the platform is build as a combination of external services including intelligent data processing services (mathematics and statistics), deep learning services and other auxiliary services and tools distributed all over the wide web to provide a powerful way for data processing.

The ontologies of the SMDA system were built using prior information and measurements received from patients diagnostics as the result of the series of experiments. The fragment of domain ontology is presented in Fig. 8.

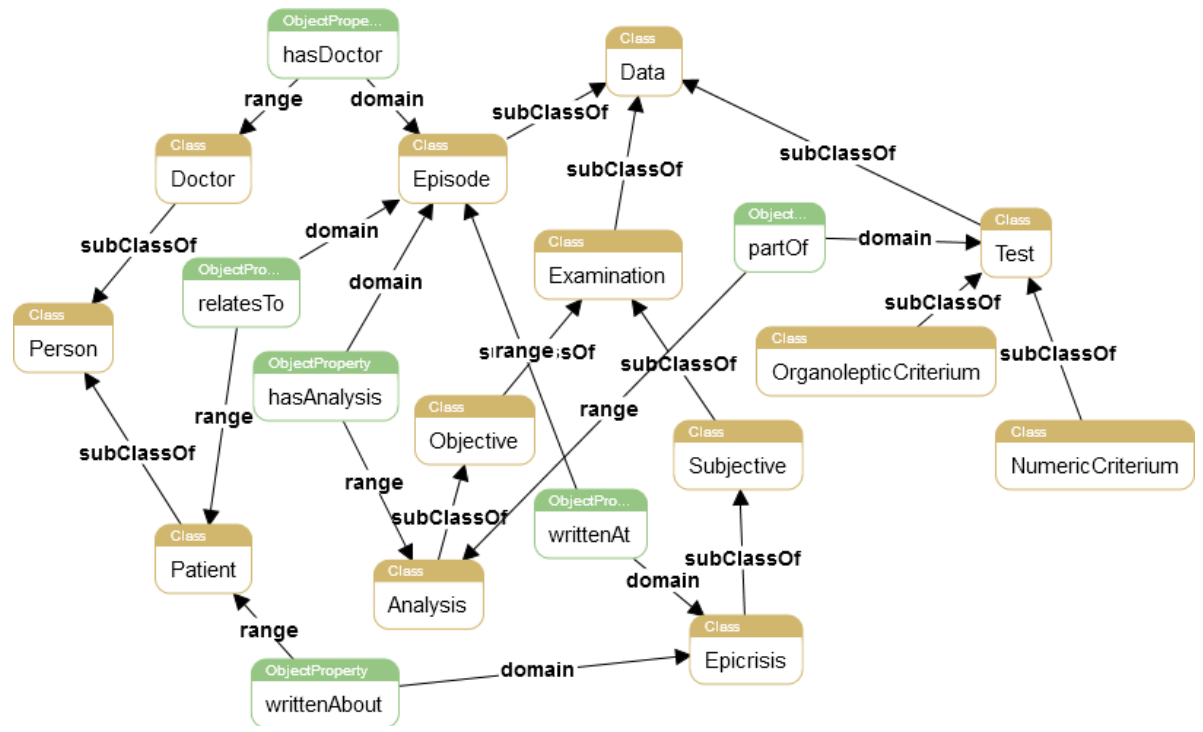

Fig. 8

The case we would like to analyze is the results of echocardiography for various diseases in order to build general template graphs for widely spread heart disorders to be able to define the disease by statistical traits specific to it. To do that, we extended ontology, analysis and diagnostics classes, in particular, with predicates specific to echocardiography method. The extended sub-hierarchy is presented in Fig. 9. The diagnostics mean extends Analysis class, thus may have a list of both numerical and organoleptic criteria (so called criteria described by natural language enumerate values). We assume that analyzing heart parameter deviations during echocardiography procedure and building a deviation template (disease model) for each disease provides us a way to assess doctor sufficiency based on comparing medical notes on the results of diagnostics and objective parameters analyzed with a help of statistically produced templates. The approach covers logical model of patient's state for the reasoning task as well as the statistical model for diagnoses.

\footnotetext{
${ }^{5} \mathrm{http} / /$ www.intersystems.com/our-products/cache/cache-overview/
} 


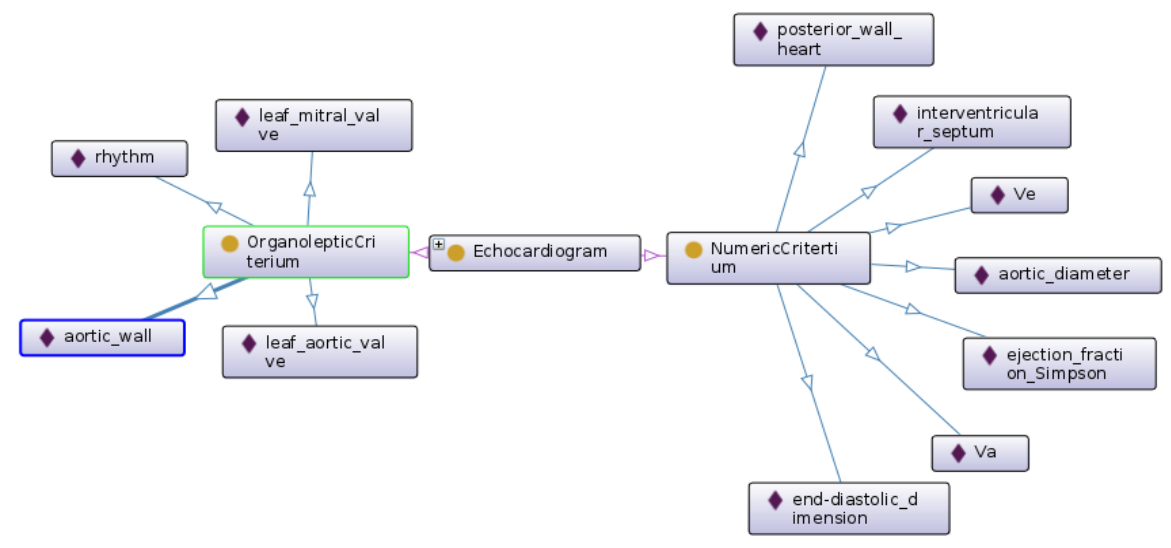

Fig. 9

Based on the analysis of patients echocardiography results during one year which includes over 10000 patients and even more echocardiography results (numerous diagnostics for some of the patients) we extracted possible tokens for parameters described by natural language tokens, an example for one of the parameters is below:

Aort valve: "without_changes", "slightly_dense", "fragmentary_calcined", "moderate_dense", "dense".

As an example, let's consider echocardiography results for 2 widely spread diagnosis: myocardial infarction and sharp coronary syndrome. A fragment of initial data is represented below:

$<<$ Date $><15.01 .2014>><$ Time $><10: 05>><<$ Time $><10: 05>><<$ Heart rate $><60>><<$ Rythm $><$ Sinusoidal $>><<$ Aorta $><$ n/a $>><<$ Aorta $><->><<$ Aortic diameter $><36>><<$ Aortic stent $><$ consolidated $>><<$ Ascending aorta $><->><<$ Left upper chamber diameter $><39>>$

Complete list includes 52 various parameters. For some parameters values can be not defined (n/a). We have investigated the influence of both organoleptic and numerical indicators for these two diagnosis.

For each disease we have calculated the deviations of the parameters values from the range defined for normal values. As the result we have identified indicators which have no deviations from the norm. These parameters are redundant parameters and we can discard them. The list of the valuable parameters can be represented either in a form of graphs or in a form of logical rules. These rules can reflect either similarities between two diagnoses or specify differences between then. An example of the graph-oriented representation is given in Fig 9. The structure of rules and their format if represented below:

if $(($ Simpson_fraction $<=60)$ or (end_diastolic_valume $>100))$ and (heartbeat $=$ "sinus") and (interatrial_septum = "without_changes") and (tricuspid_valve_leafs = "without_changes") and (!(aortic_wall = "without_changes) or !(aortic_valve_leaf = "without_canges") or !(mitral_valve_leafs = "without_chages")))

Presented constructions appear to be a valuable information source to define inference rules.

With the help of the created model a doctor with certain accuracy can define the patient disease and receive recommendation about necessary types of further analyses and treatment. The results of the models evaluation show that the accuracy of the models is about 98 - $99 \%$. But in case a doctor is interested in the diagnosis further detailing (Fig. 10) then the accuracy of the model decreases to 40$50 \%$. It means that the models need further detailing. 


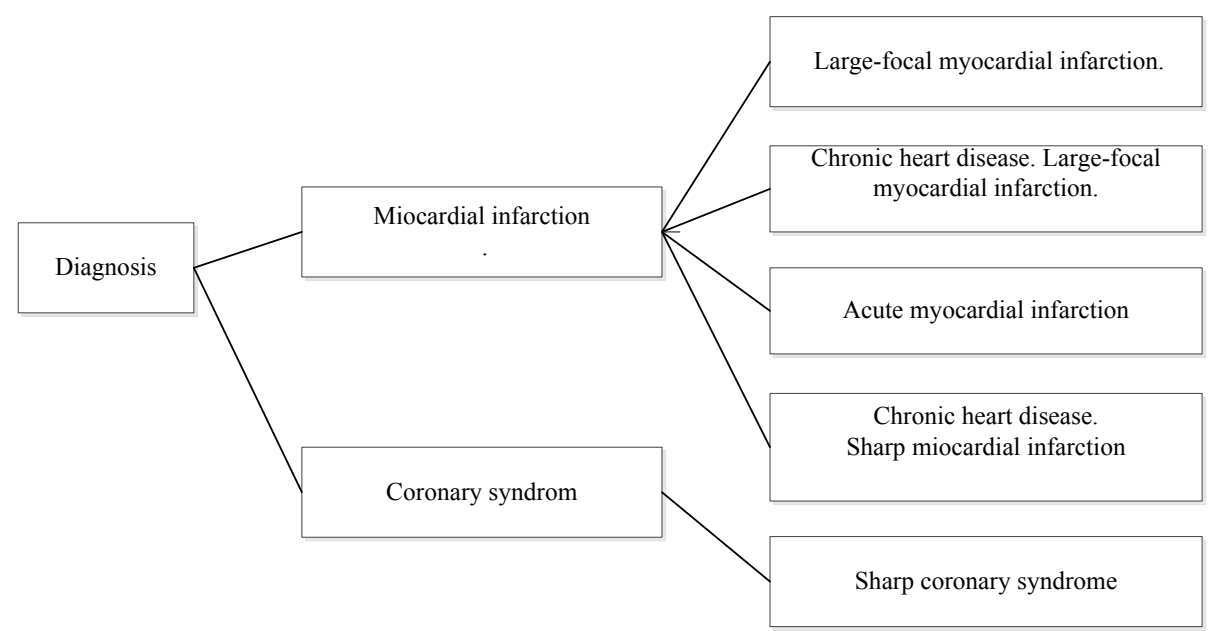

Fig. 10

One way to improve the accuracy of the models in question can be a statistical analysis of the structure of textual conclusions together with qualitative and quantitative parameters of echocardiography.

2639 cases of echocardiography were analyzed in cardiac patients in the Federal Almazov North-West Medical Research Center for the above 52 parameters and textual conclusions of cardiologists by the methods of Text Mining Statistica v.10.

The aim of the study was to search for links between echocardiographic parameters and textual conclusions of physicians to identify useful statistically significant information of the findings, improve the diagnosis and evaluate the expert value of the doctors themselves as specialists.

The following results can be used to improve the accuracy of the studied ontological models.

The concepts of words from the conclusions of doctors were singled out. These word concepts were associated with regression methods with quantitative and qualitative parameters of echocardiography.

Next, the results of the relationship of textual conclusions with quantitative parameters of echocardiography are presented. The general results are the presence of a narrow, limited, almost the same list of words, concepts and terms that describe completely different physical phenomena of a working heart in a small set, in different combinations and with different dispersions of the process.

In other words, the consciousness of a specialist in echocardiography, operating with a narrow set, a combination of medical terms, is able to describe different physical parameters of a working heart with very high predictive accuracy (see table 2,3 and fig 11. below). 
Table 2. Best words-predictors for continuous dependent variable: Diameter of aorta, Ascending aorta, Diameter of left atrium, Index of volume of the left atrium, Interventricular septum

\begin{tabular}{|c|c|c|c|c|}
\hline $\begin{array}{l}\text { Diameter of } \\
\text { aorta }\end{array}$ & Ascending aorta & $\begin{array}{c}\text { Diameter of left } \\
\text { atrium }\end{array}$ & $\begin{array}{l}\text { Index of volume } \\
\text { of the left atrium }\end{array}$ & $\begin{array}{c}\text { Interventricular } \\
\text { septum }\end{array}$ \\
\hline $\begin{array}{l}\text { cardiac is- } \\
\text { chemia } \\
\text { level } \\
\text { A0 } \\
\text { pulmonary } \\
\text { posterior } \\
\text { wall of left } \\
\text { ventricle }\end{array}$ & $\begin{array}{l}\text { cardiac ischemia } \\
\text { diastolic } \\
\text { sagging } \\
\text { breath } \\
\text { A0 } \\
\text { ascendant } \\
\text { left ventricle } \\
\text { kilogram } \\
\text { compaction } \\
\text { right atrium }\end{array}$ & $\begin{array}{l}\text { cardiac ischemia } \\
\text { right atrium } \\
\text { kilogram } \\
\text { millimeter left } \\
\text { ventricle } \\
\text { heart rate } \\
\text { sagging } \\
\text { posterior wall of } \\
\text { left ventricle } \\
\text { breath } \\
\text { diastolic }\end{array}$ & $\begin{array}{l}\text { diastolic } \\
\text { type } \\
\text { mmhg } \\
\text { cardiac ischemia } \\
\text { posterior wall of } \\
\text { left ventricle } \\
\text { right atrium } \\
\text { expanded } \\
\text { decline } \\
\text { A0 } \\
\text { mililitre }\end{array}$ & $\begin{array}{l}\text { area } \\
\text { cardiac ische- } \\
\text { mia } \\
\text { left ventricle } \\
\text { left } \\
\text { millimeter left } \\
\text { ventricle } \\
\text { sagging } \\
\text { hypertrophied } \\
\text { compaction }\end{array}$ \\
\hline
\end{tabular}

Table 3. Best words-predictors for continuous dependent variable: Ascending aorta Best predictors for continuous dependent variable: Ascending aorta (Echocardiography)

\begin{tabular}{|l|l|r|}
\hline & F-value & p-value \\
\hline Cardiac ischemia & 23,61343 & 0,000002 \\
\hline Diastolic & 12,37324 & 0,000468 \\
\hline Sagging & 11,73169 & 0,000656 \\
\hline Breath & 10,49978 & 0,001259 \\
\hline $\mathbf{a 0}$ & 10,10237 & 0,000048 \\
\hline Ascendant & 10,01684 & 0,001629 \\
\hline Left ventricle & 8,28561 & 0,004137 \\
\hline Kilogram & 7,64819 & 0,005856 \\
\hline Compaction & 7,52056 & 0,000060 \\
\hline Right atrium & 7,02160 & 0,008263 \\
\hline
\end{tabular}




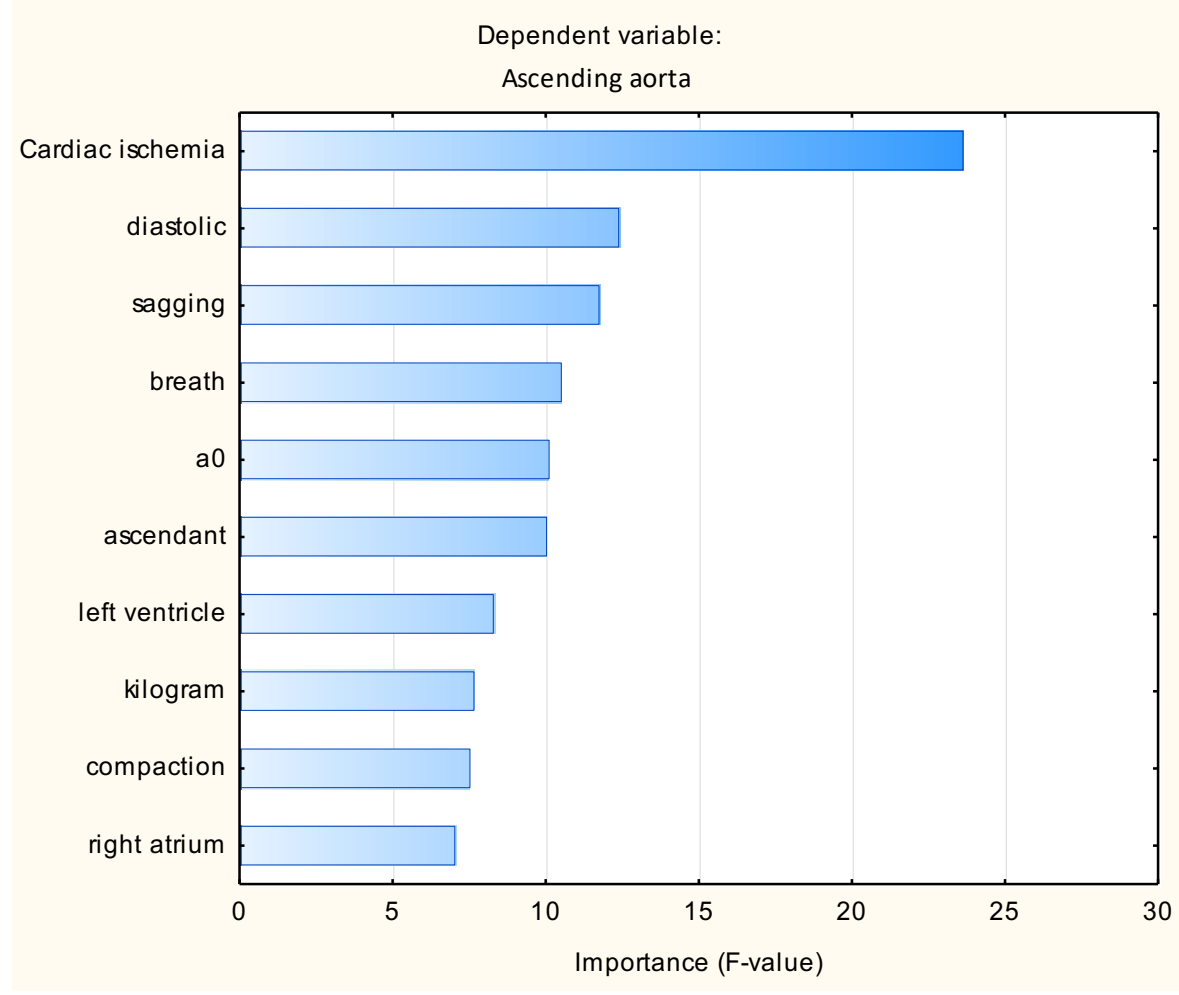

Fig. 11

\section{Conclusion}

Suggested approach can be conceded as an architectural approach to intelligent IS development. The main idea of the suggested approach is the use a combination of architectural approach to IS development and the wide usage of semantic oriented models transformation. Developed tool (Builder) allows accumulate models for future reuse.

Developed AFW was used for development a number of intelligent IS for different subject domains, including SMDA IS. This project was implemented in the Federal Almazov North-West Medical Research Centre ${ }^{6}$ (Russia) and shows the correctness of suggested models and possibility to build enough complex intelligent IS on the base of suggested models using limited human resources.

The following activities are planned for the future: i) development of new models and including them in the Builder storage; ii) integration of the Builder with popular development tools, such as tools of Eclipse Platform or NetBeans IDE; iii) extension of functionality of the developed Builder; iv) testing suggested approach for new subject domains.

Acknowledgment. The research was supported by Russian Science Foundation (project No. 17-15-01177).

${ }^{6} \mathrm{http}: / / \mathrm{www}$. almazovcentre.ru/?lang=en 


\section{References}

1. The internet of things, http://www.theinternetofthings.eu/

2. W3C. Semantic Web, http://www.w3.org/standards/semanticweb/

3. Russian Linked Culture Cloud, http://culturecloud.ru/

4. FOODpedia, http://foodpedia.tk/about?lang=ru

5. Hey J. The Data, Information, Knowledge, Wisdom Chain: The Metaphorical link. Intergovernmental Oceanographic Commission - OceanTeacher: a training system for ocean data and information management, 2004

6. International Standard ISO/IEC/IEEE 42010 Systems and software engineering. Architecture description. IEEE, 2011

7. Semantic Sensor Network XG Final Report https://www.w3.org/2005/Incubator/ssn/XGR-ssn-20110628/

8. Llinas J., Bowman C., Rogova G., Steinberg A. \& Waltz E., White F. Revisiting the JDL data fusion model II. Proceedings of the Seventh International Conference on In-formation Fusion, Stockholm, Sweden, 2004

9. Boyer J., Mili H. Agile Business Rule Development. Process, Architecture, and JRules Examples. SpringerVerlag Berlin Heidelberg 2011

10. Erl T. Service-Oriented Architecture: Analysis and Design for Services and Microservices (2nd Edition) (The Prentice Hall Service Technology Series from Thomas Erl) 2nd Edition N.Y. Prentice Hall, 2017

11. V. Yu. Osipov. Automatic Synthesis of Action Programs for Intelligent Robots, Program. Comput. Software. 42 (3) (2016) $155-160$.

12. Osipov V. Yu., Kuzenny V. V. Optimization of visual environment for spatial modeling. SPIIRAS Proceedings. 2010. Issue 1(12) pp. $235-246$.

13. Enterprise Architect User Guide, www.sparxsystems.com

14. Popovich V., Schrenk M., Korolenko K. Information Fusion and Geographic Information Systems .V.13. Proceedings of the Third International Workshop, 2007

15. http://www.almazovcentre.ru/?lang=en 